\title{
Surgical-site infections following cranial surgery: is it time to reconsider our preventive measures?
}

\author{
Parmenion P. Tsitsopoulos ${ }^{1}$
}

Received: 11 October 2018 / Accepted: 16 October 2018 / Published online: 24 October 2018

(C) Springer-Verlag GmbH Austria, part of Springer Nature 2018

Neurosurgical patients are prone to develop nosocomial infections due to their exposure to various risk factors such as impaired neurological status, long operations, stay in intensive care unit, invasive devices, and weakened immune system. As a result, defense mechanisms can be overwhelmed leading to an infectious process which can, in turn, influence negatively morbidity and mortality $[4,7]$.

Patients undergoing neurosurgery are exposed to several factors which can predispose to a surgical-site infection (SSI) [3]. Infections in the postcraniotomy period are of particular interest for neurosurgeons and include a wide spectrum of entities namely superficial and deep wound infection, meningitis, empyema and abscess, and bone flap infection $[2,6]$. In particular, those that require reoperation and prolong length of stay can have a significant negative impact on hospital care, on financial cost, and on the patient himself. Therefore, special emphasis should be placed in their prevention.

Since 2015, Jörger and coworkers have applied a discrete infection prevention bundle in their elective craniotomies. They used octenidine as antiseptic, double skin preparation was done, glue was applied instead of staples or sutures for skin closure, and cortisol was not given perioperatively. The staff was trained appropriately to ensure proper implementation of the measures. The patient sample was divided into two groups, control and study, before and after the use of the proposed infection prevention bundle, respectively. Only SSIs requiring reoperation were included in the analysis [5].

In their busy practice, the authors have eventually included 321 and 288 cases in their comparative analysis, before and after the implementation of the infection prevention bundle,

This article is part of the Topical Collection on Infection

Parmenion P. Tsitsopoulos

ptsitsopoulos@auth.gr

1 Department of Neurosurgery, Hippokratio General Hospital, Aristotle University School of Health Sciences Faculty of Medicine, Thessaloniki, Greece respectively. Before the use of the measures, $4 \%$ of the included elective craniotomy patients developed a surgical site infection that required surgical intervention. The incidence of SSI dropped to $2 \%$ following the systematic use of the prevention bundle and therefore, a reduction of $50 \%$ was noted. As expected and in agreement with previous knowledge, the commonest isolated microorganism was Staphylococcus aureus.

The results of this study provide an important message to clinical neurosurgeons. The systematic use of a relatively straightforward approach achieved a reduction of $50 \%$ in the occurrence of SSIs mandating reoperation. Although the incidence of SSI in this department of neurosurgery was already quite low, the fact that it was further decreased following the use of the prevention bundle can have numerous advantages in clinical practice such as avoidance of other systematic complications, decreased length of stay, and reduced cost.

Although its value is acknowledged, this study has numerous criticisms. Selection bias likely existed since the study sample was carefully filtered and a distinct craniotomy population was eventually analyzed. Only those that needed surgical intervention were included but there were no specified details on the type of SSI. Further, general status, prognostic factors, and co-morbidities related to the occurrence of SSI were not evaluated. The follow-up period was relatively short and especially patients with SSI without need for reoperation may have missed. It remains uncertain how much the results would differ if patient cohorts were randomized. Lastly, although an over $50 \%$ reduction of SSIs was noted, this was not statistically significant.

Although close surveillance of SSIs can identify possible factors contributing to their occurrence and the application of hygiene measures can reduce their incidence following craniotomy procedures, the role of prophylactic antibiotics should not be underestimated. Administration of antibiotics perioperatively can, by itself, lower the incidence of SSIs adding to the effect of other preventive measures such as those applied in the paper by Jörger and coworkers. The type of 
prophylactic antibiotic regimen may also play its own role not only on the incidence of SSI but on the survival as well, as it has been recently shown that cefazolin was associated with a slightly better 90 -day survival rate compared to other antibiotics [1].

Once the infection enters the bloodstream and the cerebrospinal fluid especially in susceptible patients and in areas with high antimicrobial resistance, the situation becomes more complex as prognosis of neurosurgical patients can worsen significantly [7]. Therefore, prophylactic strategies are of undisputed value. Special attention should be taken on the investigation of patients' general status and the identification of high-risk individuals where extra preventive measures should likely be implemented in order to avoid possible catastrophic consequences of SSI.

\section{References}

1. Alotaibi AF, Mekary RA, Zaidi HA, Smith TR, Pandya A (2017) Safety and efficacy of antibacterial prophylaxis after craniotomy: a decision model analysis. World Neurosurg 105:906-912 e905
2. Buffet-Bataillon S, Haegelen C, Riffaud L, Bonnaure-Mallet M, Brassier G, Cormier M (2011) Impact of surgical site infection surveillance in a neurosurgical unit. J Hosp Infect 77:352-355

3. Buggy D (2000) Can anaesthetic management influence surgicalwound healing? Lancet 356:355-357

4. Gocmez C, Celik F, Tekin R, Kamasak K, Turan Y, Palanci Y, Bozkurt F, Bozkurt M (2014) Evaluation of risk factors affecting hospital-acquired infections in the neurosurgery intensive care unit. Int J Neurosci 124:503-508

5. Jörger A, Wimmer N, Lange N, Wagner A, Janssen I, Meyer B, Shiban E (2018) The Implementation of an infection prevention bundle reduces surgical site infections following cranial surgery. Acta Neurochir In press

6. Schipmann S, Akalin E, Doods J, Ewelt C, Stummer W, Suero Molina E (2016) When the infection hits the wound: matched casecontrol study in a neurosurgical patient collective including systematic literature review and risk factors analysis. World Neurosurg 95: 178-189

7. Tsitsopoulos PP, Iosifidis E, Antachopoulos C, Anestis DM, Karantani E, Karyoti A, Papaevangelou G, Kyriazidis E, Roilides E, Tsonidis C (2016) Nosocomial bloodstream infections in neurosurgery: a 10-year analysis in a center with high antimicrobial drugresistance prevalence. Acta Neurochir 158:1647-1654 